\title{
Clinical characteristics and disease outcomes in ER+ breast cancer: a comparison between HER2+ patients treated with trastuzumab and HER2- patients
}

Shuai Li, Jiayi Wu, Ou Huang, Jianrong He, Li Zhu, Weiguo Chen, Yafen Li, Xiaosong Chen ${ }^{*}$ and Kunwei Shen ${ }^{*}$

\begin{abstract}
Background: Trastuzumab has changed the prognosis of HER2+ breast cancer. We aimed to investigate the prognosis of ER+/HER2+ patients treated with trastuzumab, thus to guide escalation endocrine treatment in ER+ breast cancer.

Methods: ER-positive early breast cancer patients operated at Ruijin Hospital between Jan. 2009 and Dec. 2017 were retrospectively included. Eligible patients were grouped as HER2-negative (HER2-neg) or HER2-positive with trastuzumab treatment (HER2-pos-T). Kaplan-Meier analysis and Cox proportional hazards model were used to compare the disease-free survival (DFS) and overall survival (OS) between these two groups.

Results: A total of 3761 patients were enrolled: 3313 in the HER2-neg group and 448 in the HER2-pos-T group. Patients in the HER2-pos-T group were associated with pre/peri-menopause, higher histological grade, LVI, higher Ki-67 level, lower ER and PR levels (all $P<0.05$ ). At a median follow-up of 62 months, 443 DFS events and 191 deaths were observed. The estimated 5-year DFS rate was $89.7 \%$ in the HER2-neg group and $90.2 \%$ in the HER2pos-T group $(P=0.185)$, respectively. Multivariable analysis demonstrated that patients in the HER2-pos-T group had a better DFS than patients in the HER2-neg group (HR 0.52, 95\% Cl: 0.37-0.73, $P<0.001$ ). The estimated 5-year OS rates were $96.0 \%$ and $96.3 \%$ in the two groups, respectively $(P=0.133)$. Multivariate analysis found that HER2-pos-T group was still associated with significantly better OS compared with the HER2-neg group (HR 0.38, 95\% Cl: 0.22$0.67, P=0.037)$.

Conclusion: ER+/HER2+ breast cancer patients treated with trastuzumab were associated with superior outcome compared with ER+/HER2- patients, indicating HER2-positivity itself may not be an adverse factor for ER+ patients in the era of trastuzumab.
\end{abstract}

Keywords: Breast cancer, Hormone receptor, HER2, Trastuzumab, Endocrine therapy, Escalation

\footnotetext{
* Correspondence: chenxiaosong0156@hotmail.com;

kwshen@medmail.com.cn

General Surgery Department, Comprehensive Breast Health Center, Ruijin Hospital, Shanghai Jiaotong University School of Medicine, 22nd Floor, 197

Ruijin Er Road, Shanghai 200025, China
}

(c) The Author(s). 2021 Open Access This article is licensed under a Creative Commons Attribution 4.0 International License, which permits use, sharing, adaptation, distribution and reproduction in any medium or format, as long as you give appropriate credit to the original author(s) and the source, provide a link to the Creative Commons licence, and indicate if changes were made. The images or other third party material in this article are included in the article's Creative Commons licence, unless indicated otherwise in a credit line to the material. If material is not included in the article's Creative Commons licence and your intended use is not permitted by statutory regulation or exceeds the permitted use, you will need to obtain permission directly from the copyright holder. To view a copy of this licence, visit http://creativecommons.org/licenses/by/4.0/ The Creative Commons Public Domain Dedication waiver (http://creativecommons.org/publicdomain/zero/1.0/) applies to the data made available in this article, unless otherwise stated in a credit line to the data. 


\section{Introduction}

Breast cancer is the most common cancer and the leading cause of death in women. More than 2,088,849 new cases of breast cancer were estimated worldwide in 2018 [1]. Breast cancer can be categorized into at least four subtypes based on the status of molecular markers for estrogen receptor (ER), progesterone receptor (PR), and human epidermal growth factor 2 (HER2) [2].

About $15-20 \%$ of patients with breast cancer have HER2-amplfied and/or overexpressed tumors and about half HER2+ tumors express hormone receptor (HR) [3]. Previous reports showed that HER2 positivity was associated with a worse prognosis before the ear of antiHER2 therapy [4-6]. In view of that, clinicians tended to recommend escalation endocrine therapy for patients with $\mathrm{HR}+/ \mathrm{HER} 2+$ disease, such as ovarian function suppression (OFS) in pre-menopausal women.

Clinical trials have demonstrated that addition of trastuzumab to standard chemotherapy in patients with HER2+ early-stage breast cancer significantly improved disease outcome [7-11], which was not influenced by HR status. Then, should ER+/HER2+ still be considered as an indicator of poor prognosis in the era of antiHER2 treatment? In current study, we aimed to investigate the prognosis of ER+/HER2+ breast cancer patients treated with trastuzumab compared with ER+/HER2patients, thus to guide us to select appropriate endocrine treatment decision making.

\section{Materials and methods \\ Patient}

Women who underwent surgery at General Surgery Department, Comprehensive Breast Health Center, Ruijin Hospital, Shanghai Jiaotong University School of Medicine from January 2009 to December 2017 were retrospectively enrolled. Clinicopathological, treatment, and follow up information were retrieved from Shanghai Jiaotong University Breast Cancer Database (SJTU$\mathrm{BCDB})$. Patients who met the following criteria were enrolled for further analysis: (1) female gender, (2) invasive breast cancer, (3) no history of prior malignancy, (4) no evidence of distant metastasis at diagnosis, (5) ER+/ HER2- breast cancer or ER+/HER2 + tumors treated with trastuzumab, and (6) complete clinical and follow-up data. Those who were diagnosed with ER+/HER2+ breast cancer but received no trastuzumab treatment were excluded. This study was reviewed and approved by the independent Ethical Committees of Ruijin Hospital, Shanghai Jiao Tong University School of Medicine.

\section{Histopathological assessments}

Tumor histo-pathologic assessment was conducted by Department of Pathology, Ruijin Hospital, Shanghai Jiaotong University School of Medicine. The methods and criteria for immunohistochemistry (IHC) assessment of ER, PR, HER2 and Ki-67 were as described in our previous reports [12, 13]. The cutoff value for ER positivity and $\mathrm{PR}$ positivity was at least $1 \%$ positive tumor cells with nuclear staining [14]. HER2 status was evaluated semi-quantitatively by IHC according to the ASCO/CAP guidelines [15-17]. Tumors with IHC HER2 2+ were further examined by fluorescence in situ hybridization (FISH), and HER2 positivity was defined as IHC HER2 $3+$ or FISH +. Ki-67 expression was scored as the percentage of positive invasive tumor cells with any nuclear staining and recorded as mean percentage of positive cells.

\section{Follow-up}

All patients were followed up by outpatient visit or call every 3 months for the first 2 years after surgery, every 6 months between the 3rd and 5th years, then annually until death. Disease-free survival (DFS) was computed from the date of surgery to the date of the following events: locoregional recurrence, contralateral breast cancer, secondary non-breast malignancy, distant recurrence at any site, and death of any cause. Overall survival (OS) was defined as the time period from the date of surgery to the date of death of any cause. Diagnosis of recurrence or metastasis was generally based on patient's radiographic images and/or pathological biopsies when accessible. Last follow-up was conducted in April 2021.

\section{Statistical analysis}

Descriptive characteristics of categorical variables were tested using chi-square test or Fisher's exact test. Multivariate logistic regression analysis was used to compare the baseline clinic-pathological features and therapies between these two groups. The estimated 5-year DFS and OS were calculated by Kaplan-Meier analysis. Cox proportional hazards regression analysis was performed to calculate independent prognostic factors in ER+/ HER2+ patients treated with Trastuzumab. All statistical procedures were performed using SPSS software, version 22.0 (SPSS Company, Chicago, IL). $P<0.05$ was considered statistically significant.

\section{Results}

\section{Patients characteristics}

Between January 2009 and December 2017, 7012 consecutive female breast cancer patients received surgery. A total of 3761 patients with early invasive breast cancer were included (Fig. 1). The study flowchart was shown in the Fig. 1. The median age was 55 (IQR 46-64) years, and 1562 patients (41.5\%) were pre/peri-menopausal at diagnosis. Tumors $>2.0 \mathrm{~cm}$ were found in 2251 patients (59.5\%), and 1346 patients (35.8\%) had positive axillary lymph nodes (ALNs). There were 796 patients (21.2\%) 


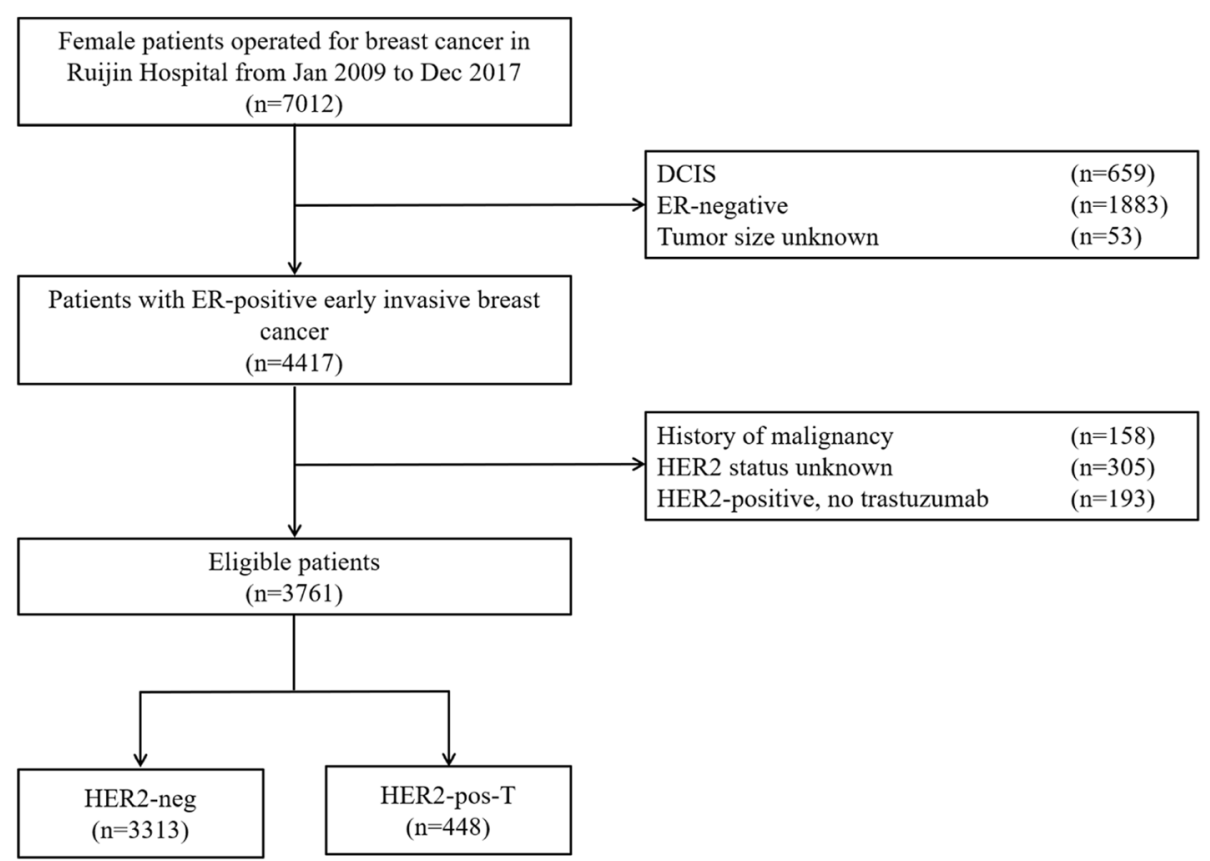

Fig. 1 Flowchart of the 3761 patients included in the study. Abbreviations: DCIS, ductal carcinoma in situ; ER, estrogen receptor; HER2, human epidermal growth factor receptor 2 .

with grade III disease and $284(7.6 \%)$ with lymphovascular invasion (LVI). All patients had ER+ disease, of whom 113 (3.0\%) had ER staining less than 10\% breast cancer cells and 3154 (83.9\%) had tumors expressing ER in more than $50 \%$ cells. PR less than $20 \%$ was found in $1671(44.4 \%)$ patients and 2016 (53.6\%) patients had Ki$67 \geq 14 \%$.

\section{Clinicopathological characteristics among ER+/HER2+ patients treated with trastuzumab}

Among the eligible patients, 448 (11.9\%) had ER+/ HER2+ tumors and received trastuzumab treatment, while the remaining 3313 (88.1\%) cases were ER+/ HER2-. Age, menstrual status, tumor size, ALN status, histological grade, pathological type, LVI status, ER level, PR level, and Ki-67 level were significantly different between the HER2-neg and HER2-pos-T groups (all $P<0.01$, Table 1). Multivariate logistic regression analysis showed that the overall distribution of menstrual status, histological grade, LVI status, ER level, PR level, and Ki-67 level had a significant difference between these two groups (Supplementary Table S1). Compared with the HER2-neg group, patients in the HER2-pos-T group were more likely to be pre/peri-menopausal (Odds ratio $[\mathrm{OR}]$ 0.68, 95\% confidence interval [CI] $0.55-0.85, P<0.001)$ and they tended to have higher histological grade (OR 1.41, 95\% CI 1.11-1.79), LVI (OR 1.67, 95\% CI 1.19-2.33, $P<0.001$ ) and higher Ki-67 level (OR 4.37, 95\% CI 3.28-5.82, $P<0.001$ ). By contrast, ER (OR 0.37, 95\% CI $0.24-0.57, P<0.001$ ) and PR (OR 0.42, 95\% CI 0.33-0.53, $P<0.001$ ) expression levels were lower in the HER2-pos-T group compared with the ER+/HER2- group.

\section{Treatment among ER+/HER2+ patients treated with trastuzumab}

Compared with the HER2-neg group, patients in the HER2-pos-T group were more likely to be treated with neo-adjuvant therapy $(11.8 \%$ versus [vs.] $5.7 \%$, $P<0.001)$ and mastectomy $(77.9 \%$ vs. $66.9 \%, P<0.001$, Supplementary Table S2). Radiotherapy was applied for $51.2 \%$ and $59.6 \%$ patients in the HER2-neg and HER2pos-T groups, respectively $(P<0.001)$. There were 91.3\% patients in the HER2-pos-T group receiving chemotherapy, which was higher than the rate in the HER2-neg group (54.0\%, $P<0.001)$. No statistically significant difference was observed in terms of adjuvant endocrine therapy between these two groups $(93.8 \%$ vs. 93.5\%, $P=0.814$ ).

\section{Prognosis of ER+/HER2+ patients treated with trastuzumab}

After a median follow-up of 62 (IQR 43-87) months, 443 DFS events were recorded (Table 2). The estimated 5-year DFS rate was $89.7 \%$ for HER2- patients and $90.2 \%$ for HER2+ patients treated with trastuzumab $(P=0.185$, Fig. 2a). Univariate analysis found that neo-adjuvant therapy, breast surgery, tumor size, ALN status, 
Table 1 Tumor and patient characteristics stratified by different groups

\begin{tabular}{|c|c|c|c|c|}
\hline Characteristics & $\begin{array}{l}\text { Total } \\
n=3761(\%)\end{array}$ & $\begin{array}{l}\text { HER2-neg } \\
n=3313 \text { (\%) }\end{array}$ & $\begin{array}{l}\text { HER2-pos-T } \\
n=448(\%)\end{array}$ & $P$ value \\
\hline Age (y/o) & 55 (46-64) & $56(46-65)$ & $51(44-59)$ & $<0.001$ \\
\hline Menstrual status & & & & $<0.001$ \\
\hline Pre/Peri- & $1562(41.5)$ & $1333(40.2)$ & $229(51.1)$ & \\
\hline Post- & 2199 (58.5) & 1980 (59.8) & $219(48.9)$ & \\
\hline Histology type & & & & $<0.001$ \\
\hline IDC & 3085 (82.0) & 2679 (80.9) & 406 (90.6) & \\
\hline Non-IDC & $676(18.0)$ & $634(19.1)$ & $42(9.4)$ & \\
\hline Histological grade & & & & $<0.001$ \\
\hline$|/| \mid$ & 2395 (63.7) & $164(65.4)$ & $231(51.6)$ & \\
\hline III & 796 (21.2) & $614(18.5)$ & 182 (40.6) & \\
\hline NA & $570(15.1)$ & $535(16.1)$ & $38(7.8)$ & \\
\hline Tumor size & & & & $<0.001$ \\
\hline$\leq 2.0 \mathrm{~cm}$ & $2251(59.5)$ & 2039 (61.5) & $212(47.3)$ & \\
\hline$>2.0 \mathrm{~cm}$ & $1510(40.1)$ & $1274(38.5)$ & $236(52.7)$ & \\
\hline ALN status & & & & 0.002 \\
\hline Negative & 2415 (64.2) & 2157 (65.1) & $258(57.6)$ & \\
\hline Positive & 1346 (35.8) & $1156(34.9)$ & $190(42.4)$ & \\
\hline LVI & & & & $<0.001$ \\
\hline No & 3477 (92.4) & 3091 (93.3) & $386(86.2)$ & \\
\hline Yes & $284(7.6)$ & $222(6.7)$ & $62(13.8)$ & \\
\hline ER & & & & $<0.001$ \\
\hline $1-9 \%$ & $113(3.0)$ & $66(2.0)$ & $47(10.5)$ & \\
\hline 10-49\% & $494(13.1)$ & $389(11.7)$ & $105(23.4)$ & \\
\hline$\geq 50 \%$ & 3154 (83.9) & 2858 (86.3) & $296(66.1)$ & \\
\hline$P R$ & & & & $<0.001$ \\
\hline$<20 \%$ & $1671(44.4)$ & $1363(41.1)$ & $308(68.8)$ & \\
\hline$\geq 20 \%$ & 2090 (55.6) & 1950 (58.9) & 140 (31.3) & \\
\hline Ki-67 & & & & $<0.001$ \\
\hline$<14 \%$ & $1745(46.4)$ & 1679 (50.7) & $66(14.7)$ & \\
\hline$\geq 14 \%$ & 2016 (53.6) & $1634(49.3)$ & $382(85.3)$ & \\
\hline
\end{tabular}

HER2, human epidermal growth factor receptor 2; BCS, breast-conserving surgery; IDC, invasive ductal carcinoma; ALN, axillary lymph node; LVI, lymphvascular invasion; ER, estrogen receptor; PR, progesterone receptor; $\mathrm{y} / \mathrm{o}$, years old

histological grade, LVI, ER level, PR level, Ki-67 level, radiotherapy, chemotherapy, and endocrine therapy were associated with DFS (all $P<0.05$, Supplementary Table S3). Neo-adjuvant therapy (HR 2.51, 95\% CI: 1.91-3.30, $P<0.001$ ), tumor $>2.0 \mathrm{~cm}$ (HR 1.54, 95\% CI: $1.26-1.88$, $P<0.001$ ), positive ALN (HR 1.58, 95\% CI: 1.29-1.93, $P<0.001$ ), grade III (HR 1.44, 95\% CI: $1.15-1.81$ ), and Ki-67 $\geq 14 \%$ (HR 1.49, 95\% CI: $1.21-1.85, P<0.001)$ were associated with worse DFS in multivariable analysis, while PR $\geq 20 \%$ (HR 0.82, 95\% CI: 0.67-1.00, $P=$ 0.050 ) and endocrine therapy (HR 0.52, 95\% CI: $0.37-$
$0.73, P<0.001)$ were associated with significantly better DFS (Table 3). More importantly, patients with HER2+ disease treated with trastuzumab had a significantly better DFS than patients in the ER+/HER2- group (HR 0.52, 95\% CI: 0.37-0.73, $P<0.001$, Table 3).

A total of 191 deaths were recorded (177 in the HER2neg group and 14 in the HER2-pos-T group, Table 2). Among these patients, $72(40.7 \%)$ in the HER2-neg group and $6(42.9 \%)$ in the HER2-pos-T group didn't have tumor recurrence. There was no significant OS difference between these two groups. The estimated 5-year OS rate was $96.0 \%$ in the HER2-neg group and $96.3 \%$ in the HER2-pos-T group $(P=0.133$, Fig. $2 b)$. Univariate analysis identified that menstrual status, neo-adjuvant therapy, breast surgery, tumor size, ALN status, histological grade, LVI, ER level, PR level, Ki-67 level, radiotherapy, and endocrine therapy were associated with OS (all $P<0.05$, Supplementary Table S3). Multivariable analysis showed that post-menopause (HR 2.33, 95\% CI: 1.69-3.22, $P<0.001$ ), neo-adjuvant therapy (HR 2.39, 95\% CI: $1.61-3.56, P<0.001)$, tumor $>2.0 \mathrm{~cm}(\mathrm{HR} 1.81$, 95\% CI: $1.32-2.48, P<0.001$ ), positive ALNs (HR 2.15, 95\% CI: $1.97-2.93, P<0.001$ ), grade III (HR 1.69, 95\% CI: $1.19-2.40$ ), and Ki-67 $\geq 14 \%$ (HR 1.43, 95\% CI: $1.02-$ 2.00, $P=0.037$ ) were associated with worse OS, while $E R \geq 50 \%$ (HR 0.44, 95\% CI: 0.24-0.78) and endocrine therapy (HR 0.46, 95\% CI: $0.30-0.71, P<0.001$ ) were associated with significantly better OS (Table 3 ). Similarly, patients in the HER2-pos-T group had significantly better OS than those in the HER2-neg group (HR 0.38, 95\% CI: $0.22-0.67, P=0.037$, Table 3).

\section{Prognostic effect of HER2 status in ER+ patients according to different subtypes}

Prognostic effect interactions were not significantly different between HER2 status and certain subgroups including age, menstrual status, tumor size, ALN status, tumor grade, ER level, PR level, and Ki-67 level (Supplementary Fig. S1).

For pre/peri-menopausal patients, the estimated 5-year DFS rate was $90.3 \%$ in the HER2-neg group and $89.6 \%$ in HER2-pos-T group $(P=0.422$, Fig. 3a). In univariate analysis, neo-adjuvant therapy, breast surgery, tumor size, ALN status, histological grade, LVI, ER level, PR level, Ki-67 level, radiotherapy, chemotherapy, and endocrine therapy were prognostic for DFS (Supplementary Table S4). As shown in the Table 4, neo-adjuvant therapy, tumor $>2.0 \mathrm{~cm}$, LVI were unfavorable prognostic factor in multivariate analysis, while endocrine therapy (HR 0.41, 95\% CI: 0.26-0.62, $P<0.001$ ) and HER2-posT (HR 0.56, 95\% CI: $0.36-0.89, P=0.013$ ) were associated with significantly better DFS. At 5 year, $97.4 \%$ patients in the HER2-neg group and 96.7\% in the HER2pos- $\mathrm{T}$ group were alive $(P=0.577$, Fig. $3 \mathrm{~b})$. Neo- 
Table 2 DFS and OS events stratified by different groups

\begin{tabular}{|c|c|c|c|}
\hline & $\begin{array}{l}\text { Overall } \\
n=3761 \text { (\%) }\end{array}$ & $\begin{array}{l}\text { HER2-neg } \\
n=3313 \text { (\%) }\end{array}$ & $\begin{array}{l}\text { HER2-pos-T } \\
n=448(\%)\end{array}$ \\
\hline \multicolumn{4}{|l|}{ DFS events } \\
\hline No recurrence & $3318(88.2)$ & $2911(87.9)$ & $407(90.8)$ \\
\hline Local-regional recurrence & $73(1.9)$ & $63(1.9)$ & $10(2.2)$ \\
\hline Contralateral breast cancer & $38(1.0)$ & $36(1.0)$ & $2(0.5)$ \\
\hline Second non-breast malignancy & $56(1.5)$ & $52(1.6)$ & $4(1.0)$ \\
\hline Distant recurrence & $198(5.3)$ & $179(5.4)$ & $19(4.2)$ \\
\hline Death without recurrence & $78(2.1)$ & $72(2.2)$ & $6(1.3)$ \\
\hline \multicolumn{4}{|l|}{ OS events } \\
\hline Alive & 3570 (94.9) & $3136(94.7)$ & $434(96.9)$ \\
\hline Death of any cause & $191(5.1)$ & $177(5.3)$ & $14(3.1)$ \\
\hline Death with recurrence & $123(3.0)$ & $105(3.1)$ & $8(1.8)$ \\
\hline Death without recurrence & $78(2.1)$ & $72(2.2)$ & $6(1.3)$ \\
\hline
\end{tabular}

HER2, human epidermal growth factor receptor 2; DFS, disease-free survival; OS, overall survival

adjuvant therapy, breast surgery, tumor size, ALN status, histological grade, LVI, ER level, PR level, and endocrine therapy affected OS in univariate analysis (Supplementary Table S4). Neo-adjuvant therapy, breast surgery, tumor size, ALN status, histological grade, and endocrine therapy remained independent prognostic factors in multivariate analysis (Table 4). After adjusting these factors, there was no obvious difference in terms of OS between these two groups (HR 0.46, 95\% CI: 0.19-1.16, $P=0.080)$.

Regarding post-menopausal patients, the estimated 5year DFS rate was $89.3 \%$ and $90.7 \%$ in the HER2-neg group and HER2-pos-T group, respectively $(P=0.292$, Fig. 3c). Univariate analysis showed that neo-adjuvant therapy, tumor size, ALN status, histological grade, ER level, PR level, Ki-67 level, radiotherapy, chemotherapy, and endocrine therapy were associated with DFS (Supplementary Table S4). As shown in Table 5, multivariate analysis suggested that neo-adjuvant therapy, tumor size, ALN status, histological grade, ER level, Ki-67 level, and endocrine therapy were independent prognostic factors for DFS. Notably, patients in the HER2-pos-T group had a significantly better DFS (HR 0.52, 95\% CI: $0.32-0.85$, $P=0.005$ ). At 5 year, there were $95.0 \%$ and $96.0 \%$ patients who were free from death in the two groups, respectively $(P=0.249$, Fig. $3 \mathrm{~d})$. Neo-adjuvant therapy, breast surgery, tumor size, ALN status, histological grade, ER level, PR level, Ki-67 level, radiotherapy, and endocrine therapy were associated with OS in univariate analysis (Supplementary Table S4). In multivariate analysis, neo-adjuvant therapy, tumor size, ALN status, histological grade, ER level, and endocrine therapy
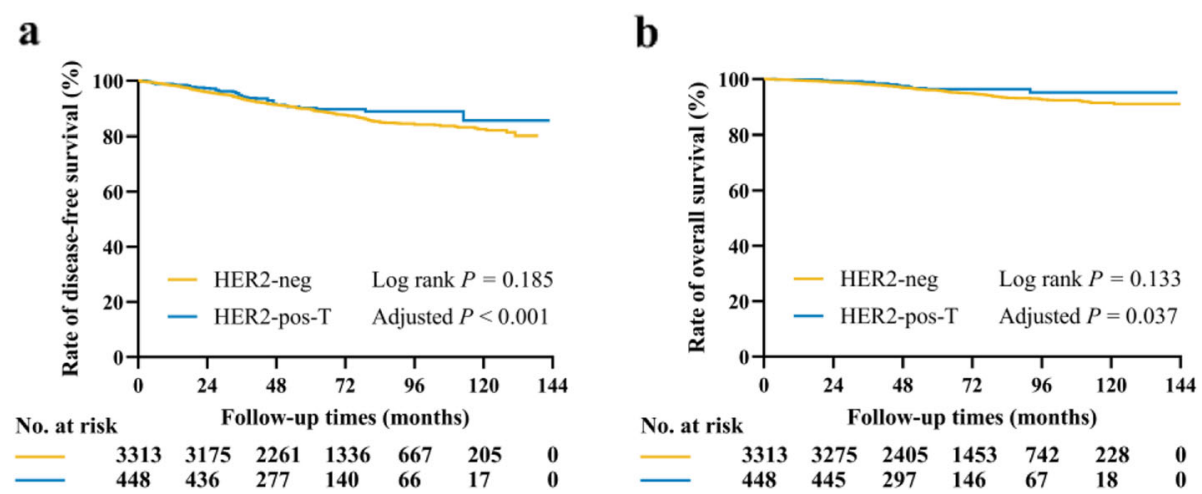

Fig. $\mathbf{2}$ Kaplan-Meier estimates of DFS and OS in the whole population. a. The estimated 5-year DFS rate was 89.7\% for HER2- patients and 90.2\% for HER2+ patients treated with trastuzumab $(P=0.185)$. HER2-pos-T was associated with significantly better DFS $(P<0.001)$ after adjusting neoadjuvant therapy, breast surgery, tumor size, ALN status, histological grade, LVI, ER level, PR level, Ki-67 level, radiotherapy, chemotherapy, and endocrine therapy. b. The estimated 5-year OS rate was 96.0\% in the HER2-neg group and 96.3\% in the HER2-pos-T group $(P=0.133)$. HER2-pos-T was associated with significantly better OS $(P=0.037)$ after adjusting menstrual status, neo-adjuvant therapy, breast surgery, tumor size, ALN status, histological grade, LVI, ER level, PR level, Ki-67 level, radiotherapy, and endocrine therapy 
Table 3 Multivariate analysis of factors associated with DFS and OS

\begin{tabular}{|c|c|c|c|c|}
\hline \multirow[t]{2}{*}{ Characteristics } & \multicolumn{2}{|l|}{ DFS } & \multicolumn{2}{|l|}{ OS } \\
\hline & HR $(95 \% \mathrm{Cl})$ & $P$ value & $\mathrm{HR}(95 \% \mathrm{Cl})$ & $P$ value \\
\hline Menstrual status (Post - vs. Pre/Peri-) & / & / & $2.33(1.69-3.22)$ & $<0.001$ \\
\hline Neo-adjuvant therapy (Yes vs. No) & $2.51(1.91-3.30)$ & $<0.001$ & $2.39(1.61-3.56)$ & $<0.001$ \\
\hline Breast surgery (BCS vs. Mastectomy) & $1.00(0.77-1.30)$ & 0.992 & $0.73(0.47-1.12)$ & 0.149 \\
\hline Tumor size $(>2.0 \mathrm{~cm}$ vs. $\leq 2.0 \mathrm{~cm})$ & $1.54(1.26-1.88)$ & $<0.001$ & $1.81(1.32-2.48)$ & $<0.001$ \\
\hline ALN status (Positive vs. Negative) & $1.58(1.29-1.93)$ & $<0.001$ & $2.15(1.57-2.93)$ & $<0.001$ \\
\hline Histological grade & & $<0.001$ & & $<0.001$ \\
\hline III vs. I/II & $1.44(1.15-1.81)$ & & $1.69(1.19-2.40)$ & \\
\hline NA vs. I/II & $1.58(1.21-2.06)$ & & $2.02(1.36-3.01)$ & \\
\hline LVI (Yes vs. No) & $1.26(0.90-1.75)$ & 0.176 & $1.55(0.94-2.54)$ & 0.084 \\
\hline ER & & 0.074 & & $<0.001$ \\
\hline $10-49 \%$ vs. $1-9 \%$ & $1.18(0.72-1.94)$ & & $0.95(0.52-1.73)$ & \\
\hline$\geq 50 \%$ vs. $1-9 \%$ & $0.89(0.55-1.45)$ & & $0.44(0.24-0.78)$ & \\
\hline PR ( $\geq 20 \%$ vs. $<20 \%)$ & $0.82(0.67-1.00)$ & 0.050 & $0.77(0.55-1.07)$ & 0.118 \\
\hline Ki-67 ( $\geq 14 \%$ vs. < 14\%) & $1.49(1.21-1.85)$ & $<0.001$ & $1.43(1.02-2.00)$ & 0.037 \\
\hline Radiotherapy (Yes vs. No) & $0.97(0.78-1.21)$ & 0.790 & $1.09(0.74-1.59)$ & 0.675 \\
\hline Chemotherapy (Yes vs. No) & $1.15(0.91-1.46)$ & 0.236 & $0.94(0.66-1.35)$ & 0.744 \\
\hline Endocrine therapy (Yes vs. No) & $0.56(0.41-0.76)$ & $<0.001$ & $0.46(0.30-0.71)$ & $<0.001$ \\
\hline Group (HER2-pos-T vs. HER2-neg) & $0.52(0.37-0.73)$ & $<0.001$ & $0.38(0.22-0.67)$ & 0.037 \\
\hline
\end{tabular}

HER2, human epidermal growth factor receptor 2; BCS, breast-conserving surgery; ALN, axillary lymph node; LVI, lymph-vascular invasion; ER, estrogen receptor; $\mathrm{PR}$, progesterone receptor; $\mathrm{y} / \mathrm{o}$, years old

remained as independent prognostic factors (Table 5). Similarly, patients with HER2+ disease and treated with trastuzumab had significantly better OS compared with patients in the HER2-neg group (HR 0.37, 95\% CI: 0.17$0.77, P=0.008$ ).

Among 243 patients who received neo-adjuvant therapy, the 5 -year DFS rate was $71.9 \%$ for those in the HER2-pos-T group and $71.3 \%$ for those in the HER2neg group $(P=0.463$, Supplementary Fig. S2a). In multivariate analysis, patients in the HER2-pos-T group had significantly superior DFS (HR 0.51, 95\% CI: 0.27-0.97, $P=0.041)$. The 5 -year OS rate was $87.7 \%$ and $85.1 \%$ for the two arms, respectively $(P=0.341$, Supplementary Fig. S2b). There was no difference between the two groups in OS (HR 0.40, 95\% CI: $0.15-1.09, P=0.073$ ).

The 5-year DFS rate was $91.9 \%$ for ER+/HER2+ patients treated with chemotherapy and trastuzumab and 87.6\% for ER+/HER2- patients who received chemotherapy $(P=0.011$, Supplementary Fig. S2c). The 5-year OS rate was $97.3 \%$ and $95.2 \%$ for the two arms, respectively $(P=0.002$, Supplementary Fig. S2d $)$. Patients in the HER2-pos- $T$ group had significantly superior DFS (HR 0.51, 95\% CI: $0.35-0.74, P<0.001)$ and OS (HR 0.32, 95\% CI: $0.17-0.63, P=0.001)$ than those in the HER2neg group among the chemotherapy subset.

For patients receiving radiotherapy, the 5-year DFS rate was $89.8 \%$ for the HER2-pos-T group and $87.9 \%$ for the HER2-neg group $(P=0.216$, Supplementary Fig. S2e). The 5-year OS rate was $96.6 \%$ and $95.4 \%$ for the two arms, respectively $(P=0.127$, Supplementary Fig. S2f). Similarly, DFS (HR 0.53, 95\% CI: $0.35-0.81, P=$ 0.003 ) and OS (HR 0.32, 95\% CI: 0.15-0.68, $P=0.003$ ) were significantly better in favor of the HER2-pos-T group.

The 5-year estimates of DFS rates were $89.7 \%$ for the HER2-pos-T group and 91.5\% for the HER2-neg group among those treated with a selective estrogen receptor modulator (SERM) $(P=0.573$, Supplementary Fig. S3a). Estimated 5-year OS rates were $96.8 \%$ and $97.7 \%$ for the two arms, respectively $(P=0.651$, Supplementary Fig. S3b). Patients in the HER2-pos-T group had significantly better DFS (HR 0.52, 95\% CI: 0.28-0.96, $P=0.038$ ) while OS (HR 0.46, 95\% CI: 0.14-1.58, $P=0.219$ ) was comparable between the two arms. For the 297 patients assigned SERM-AI, the 5-year DFS, OS rates were $95.2 \%, 97.2 \%$ for the HER2-pos-T group and $95.0 \%$, 99.1\% for the HER2-neg group, respectively $(P=0.605$, 0.228 , Supplementary Fig. S3c, d). No differences were observed in DFS (HR 0.47, 95\% CI: 0.09-2.44, $P=0.371$ ) and OS (HR 3.03, 95\% CI: 0.36-25.15, $P=0.305$ ) in multivariate analysis. Among patients receiving an AI, the DFS rate was $90.0 \%$ and $89.1 \%$ for the HER2-pos-T and HER2-neg groups $(P=0.334$, Supplementary Fig. S3e). A total of $97.7 \%$ in the HER2-pos-T group and 
$\mathbf{a}$

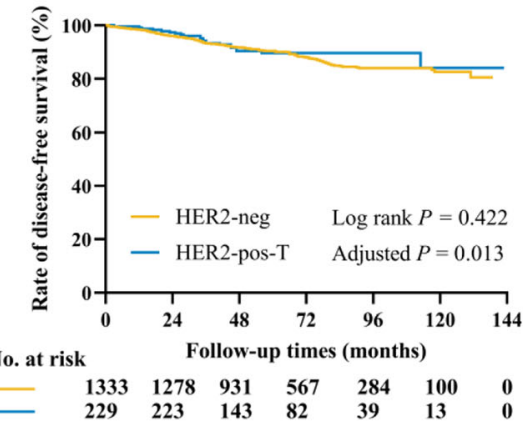

c

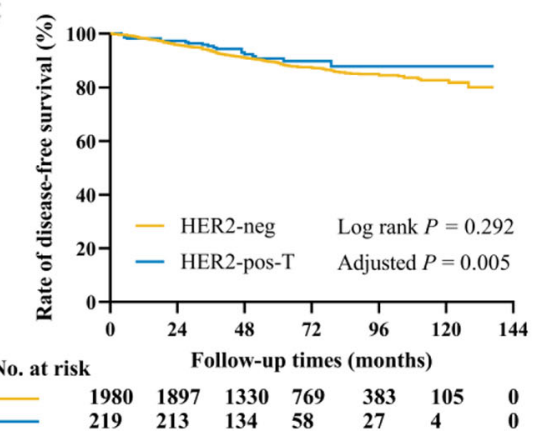

b

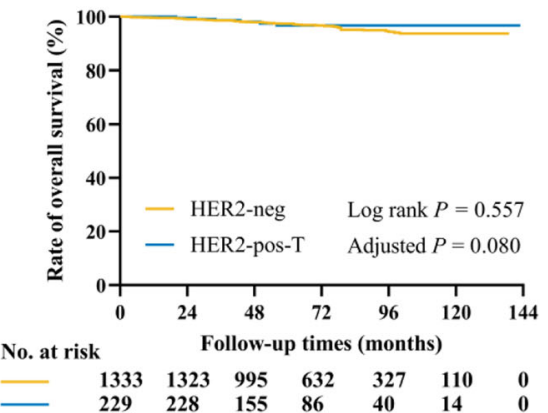

d

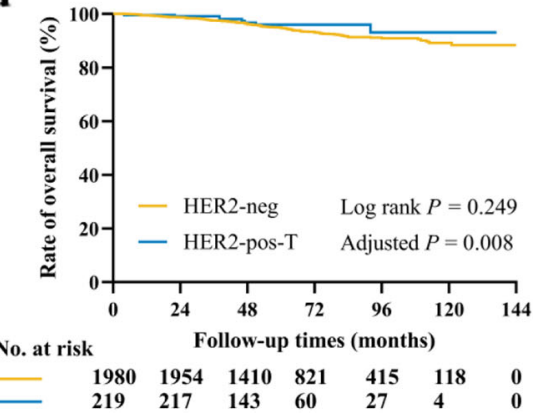

Fig. $\mathbf{3}$ Kaplan-Meier estimates of DFS and OS for pre/peri-menopausal patients ( $\mathbf{a}$ and $\mathbf{b}$ ) and post-menopausal patients (c and d). a. The estimated 5-year DFS rate was $90.3 \%$ for HER2- patients and $89.6 \%$ for HER2+ patients treated with trastuzumab $(P=0.422)$. HER2-pos- $T$ was associated with significantly better DFS in multivariate analysis $(P=0.013)$. b. The estimated 5 -year OS rate was $97.4 \%$ in the HER2-neg group and 96.7\% in the HER2-pos-T group $(P=0.557)$. No difference was observed between these two groups in multivariate analysis $(P=0.080)$. $\mathbf{c}$. The estimated 5-year DFS rate was $89.3 \%$ for HER2- patients and $90.7 \%$ for HER2+ patients treated with trastuzumab $(P=0.292)$. HER2-pos-T was associated with significantly better DFS in multivariate analysis $(P=0.005)$. $\mathbf{d}$. The estimated 5 -year OS rate was $95.0 \%$ in the HER2-neg group and 96.0\% in the HER2-pos-T group $(P=0.249)$. HER2-pos-T was associated with significantly better OS in multivariate analysis $(P=0.008)$

95.3\% in the HER2-neg group were alive at 5 years $(P=$ 0.042, Supplementary Fig. S3f). DFS (HR 0.58, 95\% CI: $0.37-0.93, P=0.023$ ) and OS (HR 0.26, 95\% CI: 0.11$0.65, P=0.004$ ) of the HER2-pos-T group were superior to that of the HER2-neg group.

\section{Predictive effect of HER2 status in ER+ patients}

There were 256 pre-menopausal women in the present cohort who were treated with OFS (75 with SERM, 16 with SERM-AI, and 165 with AI), and 1211 received endocrine therapy alone (Supplementary Table S2). The rate of OFS utilization was higher in the HER2-pos-T group than that in the HER2-neg group $(23.3 \%$ vs. $16.5 \%, P=0.016$ ). For HER2- patients, $86.5 \%$ in the OFS arm and $92.2 \%$ in the Non-OFS arm were disease-free at 5 year $(P=0.013$, Supplementary Fig. S4a). While the 5year DFS rate was $89.5 \%$ in the OFS arm and $90.2 \%$ in the Non-OFS arm among HER2+ patients $(P=0.995$, Supplementary Fig. S4b). There was no interaction between HER2 status and OFS benefit in terms of DFS $(P=0.556)$. For HER2- patients, $97.8 \%$ in the OFS arm and $98.0 \%$ in the Non-OFS arm were alive at 5 year $(P=$ 0.245 , Supplementary Fig. S4c). The rates among HER2+ patients were $100.0 \%$ and $96.3 \%$, respectively $(P=0.277$, Supplementary Fig. S4d). Similarly, no interaction between HER2 status and OFS benefit in OS were observed $(P=0.965)$.

\section{Discussion}

Patients with ER+/HER2+ breast cancer had a worse prognosis compared to those with ER+/HER2- disease in the pre-trastuzumab treatment era, who were more frequently treated with chemotherapy and escalation endocrine therapy. Consistent with previous studies, our current study found that ER+/HER2+ tumors were associated with pre/ peri-menopause, higher histological grade, LVI, higher Ki67 level, lower ER and PR levels. After adjusting for these characteristics, the DFS and OS rates were significantly better for ER+/HER2+ patients treated with trastuzumab than those with ER+/HER2- tumor, indicating HER2positivity itself may not be an adverse factor for ER+ patients if they were treated with trastuzumab, which may guide further clinical treatment decision making.

Previous reports validated that ER+/HER2+ breast cancers were associated with more unfavorable clinical features compared with ER+/HER2- diseases. A study 
Table 4 Multivariate analysis of factors associated with DFS and OS in pre/peri- menopausal patients

\begin{tabular}{|c|c|c|c|c|}
\hline \multirow[t]{2}{*}{ Characteristics } & \multicolumn{2}{|l|}{ DFS } & \multicolumn{2}{|l|}{ OS } \\
\hline & HR $(95 \% \mathrm{Cl})$ & $P$ value & $\mathrm{HR}(95 \% \mathrm{Cl})$ & $P$ value \\
\hline Neo-adjuvant therapy (Yes vs. No) & $3.74(2.56-5.46)$ & $<0.001$ & $2.27(1.22-4.21)$ & 0.009 \\
\hline Breast surgery (BCS vs. Mastectomy) & $0.84(0.59-1.21)$ & 0.841 & $0.42(0.17-1.00)$ & 0.050 \\
\hline Tumor size (> $2.0 \mathrm{~cm}$ vs. $\leq 2.0 \mathrm{~cm}$ ) & $1.84(1.34-2.51)$ & $<0.001$ & $2.89(1.46-5.71)$ & 0.002 \\
\hline ALN status (Positive vs. Negative) & $1.14(0.78-1.65)$ & 0.503 & $2.10(1.16-3.83)$ & 0.015 \\
\hline Histological grade & & 0.328 & & 0.013 \\
\hline III vs. I/II & $1.30(0.92-1.84)$ & & $2.45(1.34-4.50)$ & \\
\hline NA vs. I/II & $1.06(0.65-1.71)$ & & $1.92(0.87-4.27)$ & \\
\hline LVI (Yes vs. No) & $1.76(1.16-2.67)$ & 0.008 & $1.82(0.87-3.82)$ & 0.112 \\
\hline ER & & 0.146 & & 0.055 \\
\hline $10-49 \%$ vs. $1-9 \%$ & $0.96(0.47-1.93)$ & & $0.65(0.25-1.73)$ & \\
\hline$\geq 50 \%$ vs. $1-9 \%$ & $0.70(0.35-1.39)$ & & $0.37(0.14-0.98)$ & \\
\hline PR ( $\geq 20 \%$ vs. $<20 \%)$ & $0.86(0.62-1.20)$ & 0.384 & $0.83(0.44-1.55)$ & 0.559 \\
\hline Ki-67 ( $\geq 14 \%$ vs. $<14 \%)$ & $1.51(1.09-2.09)$ & 0.013 & / & / \\
\hline Radiotherapy (Yes vs. No) & $1.23(0.86-1.77)$ & 0.254 & / & / \\
\hline Chemotherapy (Yes vs. No) & $1.50(1.04-2.17)$ & 0.032 & / & / \\
\hline Endocrine therapy (Yes vs. No) & $0.41(0.26-0.62)$ & $<0.001$ & $0.37(0.19-0.73)$ & 0.004 \\
\hline Group (HER2-pos-T vs. HER2-neg) & $0.56(0.36-0.89)$ & 0.013 & $0.46(0.19-1.10)$ & 0.080 \\
\hline
\end{tabular}

HER2, human epidermal growth factor receptor 2; BCS, breast-conserving surgery; ALN, axillary lymph node; LVI, lymph-vascular invasion; ER, estrogen receptor; $\mathrm{PR}$, progesterone receptor; $\mathrm{y} / \mathrm{o}$, years old

Table 5 Multivariate analysis of factors associated with DFS and OS in post- menopausal patients

\begin{tabular}{|c|c|c|c|c|}
\hline \multirow[t]{2}{*}{ Characteristics } & \multicolumn{2}{|l|}{ DFS } & \multicolumn{2}{|l|}{ OS } \\
\hline & HR $(95 \% \mathrm{Cl})$ & $P$ value & HR (95\% Cl) & $P$ value \\
\hline Neo-adjuvant therapy (Yes vs. No) & $2.24(1.51-3.30)$ & $<0.001$ & $2.18(1.30-3.66)$ & 0.003 \\
\hline Breast surgery (BCS vs. Mastectomy) & / & / & $0.92(0.59-1.44)$ & 0.722 \\
\hline Tumor size (> $2.0 \mathrm{~cm}$ vs. $\leq 2.0 \mathrm{~cm}$ ) & $1.46(1.13-1.89)$ & $<0.001$ & $1.53(1.06-2.20)$ & 0.024 \\
\hline ALN status (Positive vs. Negative) & $1.74(1.34-2.26)$ & $<0.001$ & $2.23(1.55-3.19)$ & $<0.001$ \\
\hline Histological grade & & $<0.001$ & & 0.004 \\
\hline III vs. I/II & $1.54(1.13-2.09)$ & & $1.53(1.00-2.36)$ & \\
\hline NA vs. I/II & $2.05(1.48-2.85)$ & & $2.08(1.31-3.29)$ & \\
\hline ER & & 0.043 & & $<0.001$ \\
\hline $10-49 \%$ vs. $1-9 \%$ & $1.56(0.76-3.21)$ & & $1.01(0.47-2.18)$ & \\
\hline$\geq 50 \%$ vs. $1-9 \%$ & $1.02(0.51-2.02)$ & & $0.45(0.22-0.93)$ & \\
\hline PR ( $\geq 20 \%$ vs. $<20 \%)$ & $0.84(0.64-1.09)$ & 0.194 & $0.76(0.52-1.13)$ & 0.177 \\
\hline Ki-67 ( $\geq 14 \%$ vs. < 14\%) & $1.50(1.14-1.99)$ & 0.004 & $1.39(0.94-2.07)$ & 0.098 \\
\hline Radiotherapy (Yes vs. No) & $0.91(0.66-1.25)$ & 0.549 & $1.13(0.71-1.79)$ & 0.605 \\
\hline Chemotherapy (Yes vs. No) & $1.12(0.83-1.52)$ & 0.466 & / & / \\
\hline Endocrine therapy (Yes vs. No) & $0.56(0.37-0.86)$ & 0.007 & $0.53(0.31-0.91)$ & 0.021 \\
\hline Group (HER2-pos-T vs. HER2-neg) & $0.52(0.32-0.85)$ & 0.005 & $0.37(0.17-0.77)$ & 0.008 \\
\hline
\end{tabular}

HER2, human epidermal growth factor receptor 2; BCS, breast-conserving surgery; ALN, axillary lymph node; ER, estrogen receptor; PR, progesterone receptor; y/o, years old 
based on the SEER database from 2010 to 2014 revealed that age $<35$ y/o $(3.6 \%$ vs. $1.3 \%)$, tumor $>2.0 \mathrm{~cm}(48.8 \%$ vs. $36.8 \%)$, stage III/IV (21.5\% vs. $13.7 \%)$, tumor grade III (47.9\% vs. $17.4 \%)$, and less PR expression $(72.9 \%$ vs. 87.7\%) was more common in the HR+ /HER2+ subgroup compared to HR+ /HER2- patients [18]. Likewise, a meta-analysis showed that the co-expression of HER2 and $\mathrm{HR}$ was associated with age $<40$ y/o (12\% vs. $6 \%)$, tumor $>2.0 \mathrm{~cm}(53 \%$ vs. $39 \%)$, positive ALN (56\% vs. $42 \%)$, and tumor grade III (56\% vs. $29 \%$ ) compared to HR+ /HER2- patients [19]. Our results showed that ER+/HER2+ patients were significantly associated with pre/peri-menopause, higher histological grade, LVI, and higher Ki-67 level compared with ER+/HER2- patients. Moreover, ER+/HER2+ patients had lower ER and PR expression levels in our cohort, indicating ER+/HER2+ patients had unfavorable prognostic factors.

To the best of our knowledge, this is the first study comparing the prognosis of ER+/HER2+ patients treated with trastuzumab and those patients with ER+/HER2- tumors. Previously, two trials that assessed the efficacy of trastuzumab prospectively included a parallel observational cohort in which women with HER2- diseases received the same chemotherapy as did the HER2+ group. The 3-year results of the FinHer study indicated that survival free of distant disease was comparable among women with HER2+ cancer who received trastuzumab and those with HER2- cancer (HR 1.09, 95\% CI 0.52-2.29, $P=0.82$ ) [20]. In the NOAH trial, the estimated 5 -year rate of event-free survival was $58 \%$ in the HER2 + treated with trastuzumab group and $61 \%$ in the HER2- group [21]. However, because of the differences in clinic-pathological factors between HER2+ patients and those with HER2- tumors, the actual efficacy of trastuzumab on prognosis remained unknown. In the present study, ER+/HER2+ patients treated with trastuzumab had significantly better 5-year DFS (HR 0.52, 95\% CI: $0.37-0.73, P<0.001$ ) and OS (HR 0.38, 95\% CI: 0.22-0.67, $P=0.037)$ rates than those with ER+/HER2- tumor in multivariable analysis by adjusting clinicopathological factors, indicating HER2 positivity was no longer an adverse factor for ER+ patients if they were treated with trastuzumab, which may guide further clinical treatment decision making.

For pre-menopausal patients with high risk factors, adding OFS with endocrine therapy was recommended according to the SOFT and TEXT trials [22-27]. Several clinical trials showed heterogeneity of OFS treatment benefit according to HER2 status [23-27]. Data from the SOFT trial suggested that HER2+ patients received a greater DFS benefit from the addition of OFS to tamoxifen compared with HER2- patients (HR 0.41 vs. 0.83 , interaction $P=0.04$ ) [26]. However, the joint analysis of SOFT and TEXT showed that more treatment benefit with exemestane plus OFS than tamoxifen plus OFS was found in patients with
HER2- cancers (interaction $P=0.014$ ) [26]. Similarly, a statistically significant treatment benefit difference according to the HER2 status was found in the HOBOE trial and OFS plus letrozole was more effective compared with OFS plus tamoxifen in the HER2- patients [25]. In the SOFT and TEXT trials, $60.1 \%$ ER+/HER2+ patients received antiHER2 therapy. In our study, all ER+/HER2+ patients were treated with trastuzumab and they had significantly better disease outcome compared with HER2- patients, suggesting that only HER2 positivity itself may not be enough to recommend additional OFS treatment in pre-menopausal $\mathrm{ER}+/ \mathrm{HER} 2+$ patients if they were treated with trastuzumab.

In the present study, we observed a higher utilization rate of OFS among ER+/HER2+ patients treated with trastuzumab than ER+/HER2- patients. However, HER2 status seemed to provide no predictive information for OFS benefit with the presence of trastuzumab treatment. These results also suggested that HER2 positivity itself might not necessarily be an indicator for escalation endocrine therapy, which needed further research.

There were several potential limitations in this study. First, the study was retrospective and single-centered, which may lead to unavoidable bias in basic characteristics and treatment patterns between different arms. For example, $99.6 \%$ of the patients in the HER2-pos-T group received chemotherapy, whereas the percentage of chemotherapy was $55.6 \%$ in the HER2-neg group. Secondly, $136 \mathrm{ER}+/ \mathrm{HER} 2+$ patients who didn't receive trastuzumab (small tumor or patients reject to receive treatment) were excluded from the analysis. Moreover, the follow-up was still too short to clarify the effect of trastuzumab on disease outcomes, especially for ER+ disease, which needed additional follow-up.

\section{Conclusions}

Compared with ER+/HER2- breast cancers, ER+/HER2+ tumors were associated with unfavorable clinicpathological factors. However, disease outcome of ER+/ HER2+ patients treated with trastuzumab was better than those in the ER+/HER2- group, which was consistent in the pre- or post-menopausal women, indicating escalation of endocrine therapy may not be necessary for those ER+/HER2+ patients treated with trastuzumab, which warrants further clinical evaluation.

\section{Supplementary Information}

The online version contains supplementary material available at https://doi. org/10.1186/s12885-021-08555-4

\section{Additional file 1}

\section{Acknowledgements}

The authors would like to thank Ms. Yidong Du for her contribution in the management of SJTU-BCDB. We also appreciate the multidisciplinary team 
members and breast cancer specialized nurses for their great assistance in this study.

\section{Authors' contributions}

XSC and KWS carried out the study conception and design. SL performed the data analysis, study interpretation and draft the manuscript. XSC reviewed and revised the manuscript. JYW, OH, JRH, LZ, WGC, YFL, XSC and KWS participated in data collection. All authors read and approved the final manuscript.

\section{Funding}

This study was funded by the National Natural Science Foundation of China (Grant Number: 81772797). Shanghai Municipal Education

Commission—Gaofeng Clinical Medicine Grant Support (20172007); Ruijin

Hospital, Shanghai Jiao Tong University School of Medicine-"Guangci Excellent Youth Training Program" (GCQN-2017-A18). All these financial sponsors had no role in the study design, data collection, analysis or interpretation.

\section{Availability of data and materials}

The datasets used and/or analysed during the current study are available from the corresponding author on reasonable request.

\section{Declarations}

\section{Ethics approval and consent to participate}

This article does not contain any studies with human participants performed by any of the authors. All procedures performed in studies involving human participants were in accordance with the ethical standards of the institutional and/or national research committee and with the 1964 Helsink declaration and its later amendments or comparable ethical standards. This study was reviewed and approved by the independent Ethical Committees of Ruijin Hospital, Shanghai Jiao Tong University School of Medicine. Informed consent was obtained from all participants or, if participants are under 18 , from a parent and/or legal guardian.

\section{Consent for publication}

Not applicable.

\section{Competing interests}

The authors declare that they have no competing interests.

Received: 27 November 2020 Accepted: 9 July 2021

Published online: 13 July 2021

\section{References}

1. Bray F, Ferlay J, Soerjomataram I. Global cancer statistics 2018: GLOBOCAN estimates of incidence and mortality worldwide for 36 cancers in 185 countries. CA: A Cancer J Clin. 2018;68(6):394-424.

2. Waks AG, Winer EP. Breast cancer treatment: a review. JAMA. 2019;321(3): 288-300. https://doi.org/10.1001/jama.2018.19323.

3. Montemurro F, Di Cosimo S, Arpino G, et al. Human epidermal growth factor receptor 2 (HER2)-positive and hormone receptor-positive breast cancer: new insights into molecular interactions and clinical implications. Ann Oncol. 2013;24(11):2715-24. https://doi.org/10.1093/annonc/mdt287.

4. Slamon DJ, Clark GM, Wong SG. Human breast cancer correlation of the relapse and survival with amplification of her2/neu oncogene. Science. 1987;10(4785):334-40.

5. Slamon DJ, Godolphin W, Jones LA. Studies of the HER-2/neu protooncogene in human breast and ovarian cancer. Science. 1989;244(4905): 707-12. https://doi.org/10.1126/science.2470152.

6. Lisa R, Landberg G, Stal O, et al. HER2 status in hormone receptor positive premenopausal primary breast cancer adds prognostic, but not tamoxifen treatment predictive, information. Breast Cancer Res Treat. 2008;109(2):351-7.

7. Cameron D, Piccartgebhart MJ, Gelber RD, et al. 11 years' follow-up of trastuzumab after adjuvant chemotherapy in her2-positive early breast cancer: final analysis of the herceptin adjuvant (hera) trial. Lancet. 2017; 389(10075):1195-205. https://doi.org/10.1016/S0140-6736(16)32616-2.

8. Perez EA, Romond EH, Suman VJ, Jeong JH, Sledge G, Geyer CE Jr, et al. Trastuzumab plus adjuvant chemotherapy for human epidermal growth factor receptor 2-positive breast cancer: planned joint analysis of overall survival from NSABP B-31 and NCCTG N9831. J Clin Oncol. 2014;32(33): 3744-52. https://doi.org/10.1200/JCO.2014.55.5730.
9. Slamon D, Eiermann W, Robert N, et al. Adjuvant trastuzumab in her2 positive breast cancer. N Engl J Med. 2012;366(7):1273-83.

10. Jones SE, Collea R, Paul D, Sedlacek S, Favret AM, Gore I Jr, et al. Adjuvant docetaxel and cyclophosphamide plus trastuzumab in patients with HER2amplified early stage breast cancer: a single-group, open-label, phase 2 study. Lancet Oncol. 2013;14(11):1121-8. https://doi.org/10.1016/S1470-2045(13)70384-X.

11. Tolaney SM, Guo H, Pernas S, Barry WT, Dillon DA, Ritterhouse L, et al. Sevenyear follow-up analysis of adjuvant paclitaxel and trastuzumab trial for nodenegative, human epidermal growth factor receptor 2-positive breast cancer. J Clin Oncol. 2019;37(22):1868-75. https://doi.org/10.1200/JCO.19.00066.

12. Chen $X$, Sun $Y$, Mao $Y$, et al. Preoperative core needle biopsy is accurate in determining molecular subtypes in invasive breast cancer. BMC Cancer. 2013;13(1):390-6. https://doi.org/10.1186/1471-2407-13-390.

13. Tong Y, Chen $X$, Fei $X$, Lin L, Wu J, Huang O, et al. Can breast cancer patients with her2 dual-equivocal tumours be managed as her2-negative disease? Eur J Cancer. 2018;89:9-18. https://doi.org/10.1016/j.ejca.2017.10.033.

14. Hammond ME, Hayes DF, Dowsett M, et al. American Society of Clinical Oncology/ College of American Pathologists guideline recommendations for immunohistochemical testing of estrogen and progesterone receptors in breast cancer. J Clin Oncol. 2010;28(16):2784-95. https://doi.org/10.1200/JCO.2009.25.6529.

15. Wolff AC, Hammond ME, Schwartz JN, et al. American Society of Clinical Oncology/College of American Pathologists guideline recommendations for human epidermal growth factor receptor 2 testing in breast cancer. J Clin Oncol. 2007;25:118-45.

16. Wolff AC, Hammond ME, Hicks DG, et al. Recommendations for human epidermal growth factor receptor 2 testing in breast cancer: American Society of Clinical Oncology/College of American Pathologists clinical practice guideline update. J Clin Oncol. 2013;31(31):3997e4013.

17. Wolff AC, Hammond ME, Allison KH. Human epidermal growth factor receptor 2 testing in breast cancer: American Society of Clinical Oncology/ College of American Pathologists Clinical Practice Guideline focused update. J Clin Oncol. 2018;36(20):2105-22. https://doi.org/10.1200/JCO.2018.77.8738.

18. Chen K, Quan J, Yang J, Chen Z. The potential markers of endocrine resistance among HR+/HER2+ breast cancer patients. Clin Transl Oncol doi. 2019;22(4):576-84. https://doi.org/10.1007/s12094-019-02163-2.

19. Blows FM, Driver KE, Schmidt MK, Broeks A, van Leeuwen FE, Wesseling J, et al. Subtyping of breast Cancer by immunohistochemistry to investigate a relationship between subtype and short and long term survival: a collaborative analysis of data for 10,159 cases from 12 studies. PLoS Med. 2010;7(5):e1000279. https://doi.org/10.1371/journal.pmed.1000279.

20. Joensuu H, Kellokumpu-Lehtinen PL, Bono P, Alanko T, Kataja V, Asola R, et al. Adjuvant docetaxel or vinorelbine with or without trastuzumab for breast cancer. N Engl J Med. 2006;354(8):809-20. https://doi.org/10.1056/NEJMoa053028.

21. Gianni L, Eiermann W, Semiglazov V, Lluch A, Tjulandin S, Zambetti M, et al. Neoadjuvant and adjuvant trastuzumab in patients with HER2-positive locally advanced breast cancer (NOAH): follow-up of a randomised controlled superiority trial with a parallel HER2-negative cohort. Lancet Oncol. 2014;15(6):640-7. https://doi.org/10.1016/S1470-2045(14)70080-4.

22. Burstein HJ, Temin S, Anderson H, et al. Adjuvant endocrine therapy for women with hormone receptor-positive breast cancer: American Society of Clinical Oncology clinical practice guideline focused update. J Clin Oncol. 2016;34(14):1689-701. https://doi.org/10.1200/JCO.2015.65.9573.

23. Francis PA, Regan MM, Fleming GF, Láng I, Ciruelos E, Bellet M, et al. Adjuvant ovarian suppression in premenopausal breast cancer. N Engl J Med. 2015;372(5):436-46. https://doi.org/10.1056/NEJMoa1412379.

24. Pagani O, Regan MM, Walley BA, Fleming GF, Colleoni M, Láng I, et al. Adjuvant exemestane with ovarian suppression in premenopausal breast cancer. N Engl J Med. 2014:371(2):107-18. https://doi.org/10.1056/NEJMoa1404037.

25. Francis PA, Pagani O, Fleming GF, Walley BA, Colleoni M, Láng I, et al. Tailoring adjuvant endocrine therapy for premenopausal breast cancer. $\mathrm{N}$ Engl J Med. 2018;379(2):122-37. https://doi.org/10.1056/NEJMoa1803164.

26. Perrone F, Laurentiis M, Placido S, et al. Adjuvant zoledronic acid and letrozole plus ovarian function suppression in premenopausal breast cancer: HOBOE phase 3 randomised trial. Eur J Cancer. 2019;118:178-86. https//doi.org/10.1016/j.jca.2019.05.004.

27. Kim HA, Lee JW, Nam SJ, et al. Adding ovarian suppression to tamoxifen for premenopausal breast cancer: a randomized phase III trial. J Clin Oncol. 2020;38(5):434-43. https://doi.org/10.1200/JCO.19.00126.

\section{Publisher's Note}

Springer Nature remains neutral with regard to jurisdictional claims in published maps and institutional affiliations. 